\title{
TRANSVERSALIDAD DE GÉNERO E INTERSECCIONALIDAD \\ EN POLÍTICAS PÚBLICAS. UN ANÁLISIS COMPARADO \\ DE LA NORMATIVA ESTATAL Y CANARIA EN \\ MATERIA DE TRANSEXUALIDAD
}

\author{
Claudia Basterra Olives \\ claudiabasterra7@gmail.com
}

\section{RESUMEN}

La incorporación de la transversalidad de género a las políticas públicas ha supuesto la institucionalización del compromiso por elaborar políticas que contemplan el género como un factor que advierte de la producción de desigualdades entre hombres y mujeres. Sin embargo, existen otras desigualdades derivadas de cuestiones ajenas al género. Esto es lo que estudia la teoría interseccional, pretendiendo superar la transversalidad que parte de la desigualdad hombre-mujer, para atender a esos otros elementos cuya convergencia produce situaciones estructurales de exclusión. El presente artículo realiza un análisis, a partir de una perspectiva transversal e interseccional, de determinadas políticas públicas en materia de transexualidad en el ámbito estatal y en el contexto canario. Para ello se estudiarán diversos aspectos que conforman la teoría de la interseccionalidad y que servirán como criterio de análisis a fin de determinar la aplicación interseccional de las políticas objeto de estudio.

Palabras Clave: transversalidad de género, interseccionalidad, políticas públicas, equidad, transexualidad.

\section{GENDER TRANSVERSALITY AND INTERSECTIONALITY IN PUBLIC POLICIES. A COMPARATIVE ANALYSIS OF THE STATE AND CANARY ISLANDS REGULATIONS ON TRANSSEXUALITY}

\section{Abstract}

The incorporation of gender mainstreaming into public policies has led to the institutionalization of the commitment to develop policies that consider the gender factor as a factor that warns of the production of inequalities between men and women. However, there are other inequalities derived from non-gender issues. This is what intersectional theory studies, trying to overcome the transversality that starts from male-female inequality, to attend to those other elements whose convergence produces structural situations of exclusion. This article carries out an analysis, through a transversal and intersectional perspective, of certain public policies on transsexuality at the state level and in the Canarian context. To this end, various aspects that make up the theory of intersectionality will be studied and will serve as analysis criteria in order to determine the intersectional application of the policies under study.

KeYwords: gender transversality, intersectionality, public policies, equity, transsexuality.

DOI: https://doi.org/10.25145/j.clepsydra.2021.21.19

Revista Clepsydra, 21; marzo 2021, pp. 347-368; ISSN: e-2530-8424 


\section{INTRODUCCIÓN}

Los estudios de género e igualdad han acuñado infinidad de conceptos que han ayudado a entender los orígenes, causas y consecuencias de la aplicación de las teorías feministas a todos los ámbitos de la vida de las personas. En el espacio de los poderes e instituciones públicas, hay un término que quizá sea el que haya tenido más impacto en el diseño, ejecución e implementación de las políticas públicas de igualdad y LGTBI': el de la transversalidad de género o mainstreaming de género. Pero a raíz de los avances de las corrientes feministas no solo en Europa, sino en otros países y comunidades del mundo, surge, a finales de los años ochenta, un concepto que comienza a suscitar interés y que incide de forma clara en la teoría feminista, el concepto de interseccionalidad. Ya no se trata únicamente de una cuestión de igualdad, sino de diversidad y diferencias que interconectan e inciden de forma múltiple sobre una misma realidad. La combinación de la igualdad y la interseccionalidad en la aplicación de las políticas sociales, culturales, educativas, sanitarias, etc., genera herramientas potentes que contribuyen y contribuirán a construir realidades y espacios cada vez más diversos e inclusivos.

\section{NORMATIVA}

La igualdad y la transversalidad de género han sido objeto de regulación en diversos textos normativos a nivel europeo, nacional y local, cuestión que se estudiará con más profundidad en el epígrafe tercero de este artículo. No obstante, es importante resaltar las principales normas que constituyen el marco legislativo a partir del cual se desarrolla este artículo, y cuyo objeto atiende conseguir la igualdad real entre hombres y mujeres. A nivel estatal, destaca la Ley Orgánica 3/2007, de 22 de marzo, para la igualdad efectiva de mujeres y hombres, que promulga en su artículo 1 que

1. ${ }^{\circ}$ Las mujeres y los hombres son iguales en dignidad humana, e iguales en derechos y deberes. Esta Ley tiene por objeto hacer efectivo el derecho de igualdad de trato y de oportunidades entre mujeres y hombres, en particular mediante la eliminación de la discriminación de la mujer, sea cual fuere su circunstancia o condición, en cualesquiera de los ámbitos de la vida y, singularmente, en las esferas política, civil, laboral, económica, social y cultural para, en el desarrollo de los artículos 9.2 y 14 de la Constitución, alcanzar una sociedad más democrática, más justa y más solidaria.

2. A estos efectos, la Ley establece principios de actuación de los Poderes Públicos, regula derechos y deberes de las personas físicas y jurídicas, tanto públicas como 
privadas, y prevé medidas destinadas a eliminar y corregir en los sectores público y privado, toda forma de discriminación por razón de sexo.

Si se observa el acervo legislativo canario, hay que hacer referencia a la Ley 1/2010, de 26 de febrero, canaria de igualdad entre mujeres y hombres, que dispone en su artículo 1, relativo a su objeto, que

La presente ley tiene como objetivo hacer real y efectivo el derecho de igualdad de trato y oportunidades para, en el desarrollo de los artículos 9.2, 14 y 23 de la Constitución, y 5.2 y 30.2 del Estatuto de Autonomía para Canarias, seguir avanzando para lograr una sociedad más democrática, justa, solidaria e igualitaria, tanto en el ámbito público como privado. Asimismo, establecer los principios generales que han de presidir la actuación de los poderes públicos en materia de igualdad entre mujeres y hombres en todos los ámbitos de su vida, con independencia del lugar donde residan.

En relación con el objeto más concreto de este artículo, la interseccionalidad y su interrelación con la transexualidad, caben destacar tres textos normativos. A nivel estatal, la Ley 3/2007, de 15 de marzo, reguladora de la rectificación registral de la mención relativa al sexo de las personas. A nivel de la Comunidad Autónoma de Canarias, la Ley 8/2014, de 28 de octubre, de no discriminación por motivos de identidad de género y de reconocimiento de los derechos de las personas transexuales, y por último, a nivel estatal pero señalando su carácter de propuestas legislativas, la Proposición de Ley contra la discriminación por orientación sexual, identidad o expresión de género y caracteristicas sexuales, y de igualdad social de lesbianas, gais, bisexuales, transexuales, transgénero e intersexuales, así como la Proposición de Ley sobre la protección jurídica de las personas trans y el derecho a la libre determinación de la identidad sexual y expresión de género. grafes.

Todas estas normas serán objeto de estudio y análisis en los siguientes epí-

\section{EL CONCEPTO DE EQUIDAD Y SU RELACIÓN CON LA IGUALDAD}

La definición que del concepto «equidad» realiza la Real Academia Española (RAE) genera varias acepciones que, aplicadas al contexto de este trabajo, pueden guardar relación con esa equidad aplicada al ámbito de la igualdad de género. Dichas acepciones son:

2. ${ }^{\circ}$ Bondadosa templanza habitual, propensión a dejarse guiar, o a fallar, por el sentimiento de conciencia, más bien que por las prescripciones rigurosas de la justicia o por el texto terminante de la ley.

3. ${ }^{\circ}$ Justicia natural, por oposición a la letra de la ley positiva.

$4^{\circ}$ Moderación en el precio de las cosas o en las condiciones de los contratos.

5. Disposición del ánimo que mueve a dar a cada uno lo que merece (RAE, 2019). 
En base a esto, la equidad se desmarca del sentido taxativo de la norma, de esa igualdad formal, para relacionarse con la idea de justicia y de conciencia social. Esta es la conclusión a la que llegan también algunos investigadores y filósofos, al indicar que «el principio de equidad se refiere [...] a un principio de justicia, con arreglo a criterios que separan las diferencias percibidas como legítimas, de aquellas que son consideradas como ilegítimas y de las que resultan desigualdades [...]. Destacar la disfunción del principio de igualdad, conduce a reivindicar la necesidad de recurrir a otro principio de justicia que se adapte mejor a la realidad, en su diversidad» (Domínguez, Forest y Sénac pp. 46-47).

El filósofo John Rawls aboga por la equidad en la formulación de su Teoría de la Justicia. Los dos principios esenciales que definen su pensamiento son:

Primer Principio: Cada persona ha de tener un derecho igual al más amplio sistema total de libertades básicas, compatible con un sistema similar de libertad para todos. Segundo Principio: Las desigualdades económicas y sociales han de ser estructuradas de manera que sean para: a) mayor beneficio para los menos aventajados, de acuerdo con un principio de ahorro justo, y b) unido a que los cargos y las funciones sean asequibles a todos, bajo condiciones de justa igualdad de oportunidades (Rawls).

La aportación de Rawls es bastante acertada si se tiene en cuenta el objeto de este trabajo. En esta cita de 1979 alude a los «menos aventajados», a quienes, ante situaciones de desigualdad económica y social, concede un mayor beneficio, precisamente por esa condición de inferioridad. En relación con el presente trabajo, la categoría de «menos aventajados» la ocupa el colectivo LGTBI. En la mayoría de las sociedades del mundo, las personas LGTBI se sitúan, en mayor o menor medida, en una posición de inferioridad respecto a los «aventajados», es decir, la población manifiestamente heteronormativa. Y es precisamente esa inferioridad a la que Rawls atribuye una plusvalía, un valor ańadido, que se podría traducir en el reconocimiento de unas diferencias que generan diversidad, y que sitúan a estos grupos en una posición justa desde la que obtener oportunidades y revindicar su lucha.

Una vez definido el concepto de equidad, es preciso delimitar su relación con la igualdad, pues si bien son nociones relacionadas, carecen de una significación idéntica. Para ello, se atenderá a la conceptualización que realiza Thais Maingon, que establece cuatro principios ético-políticos agrupados en dos ejes: igualdad/justicia y universalidad/diversidad.

El primer eje sitúa la equidad en el plano de los espacios (los qué) relevantes para evaluar los adelantos y las bondades de la vida de las personas [...]. La equidad significa lo que es igualmente justo entre unos y otros [...]. En la igualdad lo importante es evitar ser tratado o considerado diferente, mientras que en la justicia lo relevante es lidiar con lo que de hecho nos hace diferentes. La igualdad tiene como objetivo una igualdad "entre iguales» y la justicia, una igualdad que se hace práctica real «entre desiguales» (Maingon 50).

Atendiendo al segundo eje, 
La equidad busca lo que se ajusta a las diferencias de unos y de otros y se relaciona con el reconocimiento de los sujetos y con la configuración de poderes que éstos tienen para participar en la vida social. El plano central son los sujetos (quiénes), sus condiciones de existencia y lo que éstos valoran (Maingon 50).

En esta línea, «la equidad ayuda a favorecer o a reestablecer el vínculo entre los sujetos y la sociedad, a través de una igualdad basada en el reconocimiento y en el poder que éstos tienen para participar en asuntos colectivos» (Maingon 51).

Para entender su relación con la igualdad, cabe destacar la afirmación que a este respecto realiza Maingon junto a Yolanda D’Elia, al afirmar que

La equidad y la igualdad son principios estrechamente relacionados, pero no significan lo mismo. En una sociedad verdaderamente justa, los principios de equidad e igualdad no se anulan uno al otro, ambos se aplican porque son interdependientes: ninguno es suficiente sin el otro. La equidad se asocia con oportunidades, mientras que la igualdad tiene que ver con el reconocimiento social y legal de derechos y el ejercicio de poder. En una sociedad donde las personas no se reconocen como iguales, es difícil que haya oportunidades para todos. En una sociedad de iguales donde no hay equidad, habrá una igualdad restringida porque todos somos diferentes desde el punto de vista biológico, social y cultural, y necesitamos cosas distintas en tiempos distintos (D’Elia y Maingon en García Prince 49).

Por tanto, queda clara la importancia de la diversidad como elemento y factor articulador de un principio de equidad que actúe en base a valores de justicia, solidaridad y proporcionalidad, conduciendo a una elaboración ecuánime de las políticas de igualdad. El principio de equidad es desarrollado por Lucien Jaume en el libro Les déclarations des droits de l'Homme, afirmando que «si la equidad se confronta a las leyes positivas, es porque no expresa el mismo principio de justicia que estas leyes. Las leyes positivas se basan en la igualdad aritmética, mientras que la equidad integra la diferencia y la desigualdad» (Domínguez, Forest y Sénac, 2013, p. 48).

Es importante que las políticas públicas de igualdad den un paso más allá al incorporar el principio de equidad a sus acciones, integrando las diferencias, lo que supone un gran avance para los grupos sociales más oprimidos, y en especial para el colectivo LGTBI. La entrada en la agenda política gubernamental significa un salto cualitativo en lo que se refiere a nuevos espacios desde los que reivindicar y visibilizar sus distintas y diversas realidades, esto es, desde la esfera pública, el ámbito institucional y el político. Es indudable que las políticas de igualdad constituyen un importante punto de partida para la integración de la perspectiva de género en todos los ámbitos de la sociedad, así como para la erradicación de la desigualdad entre los hombres y las mujeres. Pero es importante saber que la desigualdad no solo existe dentro del binarismo de género, sino que también afecta a otras personas por motivos distintos que las históricas diferencias culturales y biológicas masculinas y femeninas. Es el caso de las personas LGTBI, quienes experimentan discriminación y son víctimas de tratos desiguales por razones de su orientación sexual, identidad y expresión de género. La desigualdad de género entre hombres y mujeres ya se encuentra, desde hace bastante tiempo, dentro de la agenda política 
de nuestro país, y se vienen realizando políticas de igualdad al respecto. Ahora es el momento de que la célebre frase «lo personal es político» se aplique a las realidades LGTBI y obtenga así la protección política y legislativa que merecen en base a criterios de equidad y justicia.

\section{LA TRANSVERSALIDAD DE GÉNERO O MAINSTREAMING}

La aproximación hacia la transversalidad de género como enfoque susceptible de ser incorporado en las políticas públicas, así como la legitimación de la noción de mainstreaming, se origina en las Conferencias Mundiales sobre las Mujeres organizadas por Naciones Unidas. Así, «el concepto de aproximación integrada de la igualdad entre mujeres y hombres apareció por primera vez en los textos internacionales como resultado de la Tercera Conferencia Mundial de Naciones Unidas sobre las Mujeres», celebrada en Nairobi en 1985 (Domínguez, Forest y Sénac 233).

El concepto de enfoque integrado fue aprobado en la Conferencia Mundial de la Mujer en el año 1995 en Beijing. Lo establecido en esta Conferencia fue recogido en la Plataforma de Acción de la IV Conferencia de la Mujer, en la que se definieron acciones para integrar la perspectiva de género, destacando los artículos 57 y 76 b) de la Declaración:

El éxito de las políticas y de las medidas destinadas a respaldar o reforzar la promoción de la igualdad de género y la mejora de la condición de la mujer debe basarse en la integración de una perspectiva de género en las políticas generales relacionadas con todas las esferas de la sociedad, así como en la aplicación de medidas positivas con ayuda institucional y financiera adecuada en todos los niveles (art. 57 Declaración y Plataforma de Acción de Beijing, 1995).

El concepto de mainstreaming fue definido por el Programa de Acción Comunitaria de la UE para la igualdad de oportunidades entre hombres y mujeres (1996-2000) como la «[...] integración de la igualdad de oportunidades entre mujeres y hombres en la elaboración, ejecución y seguimiento de todas las políticas y acciones de la Unión Europea y de los estados miembros, dentro del respeto de sus respectivas competencias» (art. 2 Dec. No 95/593/CE del Consejo Europeo, de 22 de diciembre de 1995).

Otras normas e instrumentos de carácter comunitario como el Tratado de Ámsterdam (1997), la Agenda Social Europea (Lisboa, 2001) y la Directiva 2006/54/ $\mathrm{CE}$, se hacen eco e incorporan la transversalidad como principio a tener en cuenta en la formulación de políticas y acciones públicas (Instituto Andaluz de la Mujer, 2010).

En el ámbito estatal, destaca el Plan Estratégico de Igualdad de Oportunidades (2008-2011), entre cuyos principios rectores se encuentra el de Transversalidad de la perspectiva de género, entendida como una «herramienta que busca modificar las formas actuales de la política, de modo que se tomen como referencia las experiencias, las aportaciones de las mujeres, su modo de estar en el mundo y su conocimiento» (PEIO 2008-2011). 
Por lo que respecta a la Ley estatal en materia de igualdad por excelencia, la Ley Orgánica 3/2007, la transversalidad de género se contempla en el artículo 15 y vincula a los poderes públicos. Cabe destacar que, además, esta ley invoca a la transversalidad en todos los ámbitos de las políticas sectoriales como principio a tener en cuenta, como por ejemplo en la política sanitaria, cultural, educativa, ordenación territorial y vivienda, mercado laboral, etc. (Instituto Andaluz de la Mujer, 2010).

Dentro de nuestro contexto autonómico, es importante destacar, además de la Ley 1/2010, la Estrategia para la Igualdad de Mujeres y Hombres 2013-2020 de Canarias, que, entre otras cuestiones, recoge también la aplicación de la perspectiva de género de forma sectorial, estableciendo que

resulta necesario seguir profundizando y avanzando en la incorporación de la perspectiva de género en las políticas públicas. Es necesario que las políticas de igualdad giren en torno a ciertos planteamientos o premisas básicas: la transversalidad, como eje vertebrador de las distintas políticas sectoriales, de modo que la perspectiva de género esté presente en toda la acción de gobierno (EIMYHC 2013-2020).

Hasta ahora se ha mencionado la transversalidad de género aplicada a las políticas públicas de «igualdad» stricto sensu. Es interesante partir de la concepción de que la igualdad también puede -y debe- ser entendida en términos de equidad y justicia social. Por tanto, resulta coherente pensar que desde una conciencia equitativa que albergue la diversidad en lugar de excluirla, sea posible construir políticas públicas LGTBI que integren la perspectiva de género con un enfoque interseccional, para combatir no solo la desigualdad por razón de género, sino la desigualdad producida por otros factores que también producen discriminación. Este planteamiento se apoya en la existencia de estudios que responden a la cuestión de si la interseccionalidad es tomada en consideración como elemento central en la formulación de políticas de igualdad. Uno de estos estudios fue realizado por Lucas Platero en el ańo 2012, donde afirma que «el estudio de la sexualidad no normativa en el seno de las políticas de igualdad de género aparece en un segundo plano frente a aquellas cuestiones tratadas como principales» (Platero Son las políticas, 135).

Además, establece que "se parte de la premisa que algunos problemas en la implementación del principio de mainstreaming son debidos a que no se reconoce que existen múltiples interpretaciones de la (des)igualdad de género y que dicha (des)igualdad se enmarca de diferentes maneras como problema político» (Bustelo y Lombardo, 2003-2005. MAGEEQ). Legislar sobre asuntos que afectan a grupos y colectivos «localizados», «vulnerables» o «excluidos» no significa diseñar políticas interseccionales. Es por ello por lo que Platero afirma que

Las políticas públicas del Estado espańol abordan las desigualdades con una perspectiva monofocal o descriptiva de cierta diversidad, y se dirigen exclusivamente a la desigualdad de género o a las mujeres, la discapacidad, la familia, la juventud e infancia, los mayores, la exclusión social, el empleo, etc., y a menudo reciben el nombre de políticas sectoriales (Son las políticas, 138). 
Todo apunta a que la transversalidad de género, en su integración en las políticas públicas, no lo hace aún con un enfoque interseccional que entienda la coincidencia de factores no como simples opresiones aisladas, sino como una cuestión más compleja cuya regulación debe desmarcarse de la política sectorial para comenzar a crear políticas interseccionales.

\section{INTERSECCIONALIDAD. CONCEPTO Y APORTACIONES TEÓRICAS}

A partir de los años setenta comienzan a realizarse estudios e investigaciones sobre la interrelación de diferentes tipos de desigualdades en Estados Unidos, coincidiendo con el momento en que el feminismo negro y chicano puso de manifiesto los efectos simultáneos de discriminación que se generaban en torno a la raza, el género y la clase social (Cubillos 121).

Algunas autoras feministas americanas como Angela Davis, Moraga y Anzaldúa o Bell Hooks «manifestaron y analizaron la diversidad del grupo mujeres y cómo sus experiencias de desigualdad social y política se construían tanto en función de la desigualdad de género como de la raza, la clase social y/o la orientación sexual» (Cruells 34). Sin embargo, es en el año 1989 cuando se acuña de manera académica y oficial el término «interseccionalidad», a través de un artículo publicado por la jurista Kimberlé Crenshaw, definiéndolo como «aquel que indica la relación y articulación entre las desigualdades sociales» (Cruells 34). De esta forma, el término «cristaliza un debate sobre las dificultades de formular y conceptualizar la dinámica de las relaciones sociales, tanto en la acción pública como colectiva» (Domínguez, Forest y Sénac 144).

Se podría decir que uno de los factores clave para que surgiera la teoría de la interseccionalidad fue la evidencia - por parte del movimiento feminista-, de la política de la identidad impuesta por el ideario moderno. Es decir, un sistema que se desarrolla en torno a un elemento central masculino, occidental, blanco, heterosexual y de clase media-alta, que ocupa una posición de preeminencia y que, por ende, determina la existencia de otras identidades y otros sujetos, que conviven en los márgenes de ese sistema patriarcal hegemónico. Dentro del feminismo, esta diferenciación hombre-centro y mujer-margen, puede extrapolarse a que el «feminismo hombre» sería el feminismo blanco o hegemónico, mientras que el «feminismo mujer» serían todos los feminismos negros, lésbicos, indígenas o queer.

Desde la década de los ochenta hasta la actualidad, se han realizado numerosos análisis e investigaciones sobre este término, con el objetivo de aportar nuevos enfoques a fin de conformar una teoría de la interseccionalidad consolidada. Cabe destacar en este punto las contribuciones pioneras de Crenshaw, quien distingue en su teoría dos tipos de interseccionalidad: la estructural, en la que hace referencia a diversos factores que interactúan entre sí y que son los que provocan esa desigualdad de forma interseccional, y la política, poniendo el foco en aquellas realidades que se sitúan en el centro de la agenda pública y política y que, por ende, suponen la marginalización de otras. 
Otras aportaciones del término interseccionalidad las realiza Patricia Hill Collins, que acuña en su teoría el concepto de «matriz de dominación» para explicar la interdependencia de los diferentes ejes de opresión. Leslie McCall, por su parte, aporta tres enfoques destinados a deconstruir esas categorías que precisamente causan opresión (anticategórico), a entender la complejidad de la desigualdad dentro de un grupo concreto (intracategórico), así como utilizar esas categorías como elementos de análisis (intercategórico). Es interesante la definición que realiza Sirma Bilge, quien entiende que la interseccionalidad debe comprenderse desde un enfoque integrado sin diferenciación entre los ejes de opresión que pueden interrelacionar.

El estudio de la igualdad, de su alcance y de todo lo que conlleva su aplicación en una sociedad ya es una tarea compleja que requiere de un estudio en profundidad. No obstante, considero que es interesante incorporar la interseccionalidad como otro paradigma desde el que interpretar la igualdad y la aplicación de la transversalidad de género en las políticas públicas. La interseccionalidad no solo debe estar presente en la perspectiva de quien analiza, sino también en el imaginario y en los conocimientos de quien diseńa y elabora los instrumentos de política pública. De nada sirve que se analice una norma, un plan de igualdad o medidas de acción positiva desde un enfoque integrado de las desigualdades, si quien decide sobre su contenido no posee la formación ni la convicción necesarias para plasmar en su redacción este enfoque multifactorial. De igual forma que se prevé la aplicación de la transversalidad de género en diferentes ámbitos (social, educativo, económico, laboral, cultural, etc.), es necesario que dichos poderes tengan en cuenta, en el desempeño de sus funciones y en la construcción de sus políticas, el factor de la diversidad, así como la existencia de múltiples factores discriminatorios que se relacionan entre sí provocando situaciones de exclusión. Por esto, es importante que las políticas públicas sean transversales y también interseccionales, tratando las diferentes desigualdades no como supuestos separados de "doble o triple exclusión», sino como lo que son, el resultado de un engranaje de opresiones estructurales relacionadas cuya concurrencia genera situaciones graves de discriminación.

Con el objetivo de observar el carácter interseccional o no de una política pública, las teorías y enfoques expuestos anteriormente serán tomados como criterios de análisis de tres leyes concretas: la Ley 3/2007, de 15 de marzo, reguladora de la rectificación registral de la mención relativa al sexo de las personas, la Ley 8/2014, de 28 de octubre, de no discriminación por motivos de identidad de género y de reconocimiento de los derechos de las personas transexuales, y la Proposición de Ley contra la discriminación por orientación sexual, identidad o expresión de género y caracteristicas sexuales, y de igualdad social de lesbianas, gais, bisexuales, transexuales, transgénero e intersexuales (Proposición de Ley LGTBI). 


\section{ANÁLISIS DE LA APLICACIÓN INTERSECCIONAL DE LAS LEYES}

El primer criterio de análisis responde a los tipos de interseccionalidad que diferenciaba Crenshaw. La Ley 3/2007, reguladora de la rectificación registral, podría encajar en la crítica que Crenshaw realiza a las «estrategias políticas que se centran en una sola categoría, por no haber sido capaces de dar cuenta de la heterogeneidad interna de los grupos sociales» (Cruells 36), ya que se centra únicamente en una dimensión de desigualdad, la transexualidad, y, dentro de esta, en un aspecto concreto: la modificación registral del sexo. En el caso de la ley 8/2014, ocurre lo contrario, ya que a priori, la norma no se centra únicamente y de manera rígida en el eje de desigualdad "transexualidad", sino que lo hace desde la heterogeneidad al dar cuenta de que "existe una diversidad de comportamientos y respuestas entre las propias personas transexuales» (Ley 8/2014). Además, destaca la existencia de otros factores que pueden producir desigualdad como el acoso escolar, la insularidad y el desempleo.

En relación con los tres enfoques de McCall, la Ley 3/2007 no cumple con ninguno de ellos. Lejos de evitar categorizaciones, y por tanto cumplir con el enfoque anticategórico, la Ley 3/2007 se desarrolla a partir de un concepto clave, la transexualidad, definiéndola en su breve exposición de motivos como «un cambio en la identidad de género ampliamente estudiada ya por la medicina y por la psicología» (Ley 3/2007 reguladora de la rectificación registral de la mención relativa al sexo de las personas). El enfoque intracategórico tampoco existe, pues la norma no repara en la complejidad de esta realidad ni en otras categorías que inciden en ella. Cabe destacar que, aun tratándose de la primera regulación a nivel estatal de la transexualidad, no lo hace abordando esta condición de forma integral, sino que se centra en la cuestión registral del sexo como principal y único "problema» que precisa de atención política. Como consecuencia de ese segundo enfoque, el aspecto intercategórico tampoco se da, puesto que no existen diferentes categorías de análisis que permitan analizar la desigualdad entre diversos grupos como podrían ser las personas trans* atravesadas por otros factores, como ser menor, migrante, racializada/o, o intersexual.

Por el contrario, la Ley 8/2014 cumple dos de los tres enfoques de McCall, aunque con matices. El enfoque anticategórico no tiene aplicación en esta norma, pues articula todo su contenido en torno al concepto «transexualidad». Precisamente, la categorización y la delimitación conceptual de esta realidad es lo que hace posible la elaboración de una ley destinada específicamente a regular aspectos que afectan a la vida personal y social de este grupo. El enfoque intracategórico, por el contrario, sí se observa, pues se evidencian las numerosas desigualdades a nivel social, educativo, o laboral que se derivan de la pertenencia a un grupo social determinado como es el colectivo trans*. El último enfoque de este grupo, el intercategórico, es de aplicación imprecisa, pues en la exposición de motivos se tienen en cuenta variables como la insularidad, la clase social y el desempleo, solamente nombradas con relación a las realidades trans*, distanciándose de la idea de «analizar la desigualdad entre los grupos sociales» (Cruells 39), ya que la ley no los trata como grupos en sí mismos, sino como circunstancias que interactúan entre sí. 
En el caso de la Proposición de Ley LGTBI, el enfoque anticategórico tampoco se cumple, pues lejos de deconstruir las categorías de análisis relacionadas con la sexualidad y la identidad de género, este texto se elabora para garantizar la protección de grupos perfectamente delimitados como son las lesbianas, gais, bisexuales, transexuales, transgénero e intersexuales. Por tanto, se observa que el establecimiento de categorías e identidades que surgen de la categorización de un grupo que vive fuera de los límites de lo normativo es necesario en este caso para poder desarrollar una política pública que les otorgue protección y condene las discriminaciones. El enfoque intracategórico sí se da, en tanto que trata las desigualdades derivadas de la pertenencia a un grupo oprimido como es el colectivo LGTBI, agrupándolas bajo el paraguas LGTBI, pero sin tratarlas de forma separada en función de las características específicas que como subgrupos presentan -existen diferencias entre lesbianas y gais que residen en cuestiones de género a pesar de soportar una opresión similar en términos generales-. Por tanto, no se cumple con el tercer enfoque propuesto por McCall, el intercategórico.

Como tercer criterio se presenta el tipo de modelo interseccional surgido como consecuencia de la implementación del modelo europeo en los estados miembros, pudiendo ser jerárquico, integrado y estratificado. Así, la Ley 3/3007 podría responder al modelo jerárquico. La categoría que adquiere mayor prioridad es la transexualidad, si bien en la ley no consta la existencia de instituciones específicas que se deriven de la misma. Por su parte, la Ley 8/2014 responde al modelo jerárquico, pues la categoría prioritaria sobre la que trata es la transexualidad, si bien recoge otros factores susceptibles de producir discriminación como la insularidad, el desempleo o la juventud, pero que carecen de la entidad suficiente como para identificarse con un modelo integrado o estratificado. El modelo al que respondería la Proposición de Ley LGTBI sería el estratificado, pues el texto combina el tratamiento de diversas desigualdades -no solo las LGTBI, sino otras relativas a la diversidad funcional, a la población mayor, a la violencia de género, etc.-, y mantiene a su vez estructuras diferenciadas para la desigualdad central de la que se ocupa esta Proposición de Ley, que es la del colectivo LGTBI.

En cuanto al modelo de política de igualdad, pero aplicándose en este caso a las políticas LGTBI, pueden derivar los modelos single issue, discriminación múltiple e interseccional. Las políticas single issue "ponen el foco en una problemática muy concreta y pueden conseguir grandes avances en la consecución de la igualdad de los grupos destinatarios [...]. Este ha sido el caso de las políticas de igualdad mujer/ hombre [...]. En el ámbito LGTB, son muy recientes y su implementación es incipiente en el contexto europeo» (Coll-Planas). Por su parte, el modelo de discriminación múltiple entiende que "las personas que agrupa en su seno no están solamente definidas por su identidad de género y sexualidad, sino que en la configuración de su posición social intervienen otros factores, como la edad, la raza o la clase social [...] (Cruells y Coll-Planas 156).

En relación con el enfoque interseccional,

Establece el reto de abordar la intersección entre desigualdades de una forma más compleja que el modelo de la discriminación múltiple [...]. Aplicar una perspec- 
tiva interseccional en las políticas públicas se presenta como la clave para alcanzar una mayor inclusión en la medida en que muchos más grupos sociales y problemas políticos pueden ser contemplados (157).

La Ley 3/2007 adopta el modelo single issue, en tanto que se centra en una única problemática, la rectificación registral del sexo de las personas transexuales. En cuanto a la Ley 8/2014, se podría decir que posee aspectos que le hacen pivotar entre los modelos estudiados, aunque con matices: el de discriminación múltiple y el interseccional -en este último de forma imprecisa-. En primer lugar, existen varios puntos a lo largo de la norma que configuran el modelo de discriminación múltiple. A pesar de que la realidad trans* sea la causa y el objetivo principal de la ley, es cierto que se manifiestan otro tipo de factores que intervienen. Sin embargo, la ley no los trata al mismo nivel que la transexualidad, pues los nombra en artículos concretos, pero no los incluye de forma interrelacionada, por tanto, se trataría de un modelo de discriminación múltiple «a medias» que está a caballo entre el single issue y el enfoque de discriminación múltiple propiamente dicho. Ejemplo de la inclusión de otros factores, se encuentran los mecanismos de empleabilidad previstos especialmente para aquellas personas que "por su condición de joven, de mujer o de desempleado/a de larga duración, se encuentran en riesgo de padecer múltiples situaciones de discriminación» (Ley 8/2014, art. 13.b).

Otro factor es el envejecimiento que afecta a una parte de población trans*, realidad de la que la ley se hace eco al establecer el derecho de estas personas a

Recibir una protección y una atención integral para la promoción de su autonomía personal y del envejecimiento activo, que les permita una vida digna e independiente y su bienestar social e individual, así como a acceder a una atención gerontológica adecuada a sus necesidades (Ley 8/2014, art. 16.1).

El último factor de opresión que se observa en esta ley es el de condición de víctima de violencia de género. Así, se prevé un artículo destinado específicamente a la atención a mujeres transexuales víctimas de violencia de género, estableciendo que, en este caso, "tendrá acceso, en condiciones de igualdad, a los recursos asistenciales dispuestos a tal efecto por la Comunidad Autónoma de Canarias» (Ley 8/2014, art. 16 bis).

El modelo interseccional, en relación con la configuración de esta norma, tendría una aplicación, a mi juicio, incompleta. La inclusión de otros ejes de desigualdad -expuestos en párrafos anteriores-, como aspectos susceptibles de ser regulados en su interferencia con la transexualidad, originan un tratamiento simultáneo de varias desigualdades, sin que ello suponga la aplicación de una perspectiva plenamente interseccional. Tal y como establecían Cruells y Coll-Planas, se trata de contemplar muchos más grupos sociales y problemas políticos para que exista una mayor inclusión. En base a esto, la ley contempla otros problemas que afectan a la realidad de las personas trans* de carácter laboral, educativo o social, pero no integra de forma completa e interseccional otras realidades y/o grupos sociales. Como grupos sociales, alude someramente a las poblaciones jóvenes y a las mujeres trans*, y se detiene un poco más en las poblaciones de la tercera edad, para dedicar en última 
instancia un pequeño artículo a las mujeres transexuales en situación de violencia de género. Es cierto que se incluyen otras realidades además de la transexualidad que a su vez interseccionan con ella, sin embargo, el tratamiento que de ellas se realiza es puntual, a la vez que quedan muchos otros factores por incluir como la raza, la orientación sexual, la procedencia geográfica, la discapacidad, la religión, el idioma, la condición de migrante/indígena/refugiado, etc. Por tanto, no puede determinarse en términos absolutos que esta ley tenga una perspectiva interseccional entendida desde el tratamiento estructural y complejo de las desigualdades.

Con respecto a la Proposición de Ley, responde al modelo de discriminación múltiple, pues ya desde el inicio del texto, se pone de manifiesto que, además de la condición LGTBI, el colectivo se ve afectado por otros factores que en la mayoría de los casos originan situaciones graves de discriminación. No se puede afirmar que se trate de un modelo propiamente interseccional, pues la futura norma no regularía la interacción de diferentes desigualdades independientes pero vinculadas de forma estructural como pudieran ser la diversidad funcional, la migración o la etnia, sino que vincula a la desigualdad "principal» (el colectivo LGTBI) otros factores que pueden derivar de esa misma desigualdad (personas mayores LGTBI, mujeres transexuales en situación de violencia de género, asistencia sanitaria sexorreproductiva a mujeres lesbianas y bisexuales, etc.).

La definición del target es el último criterio aplicable, siendo, en el caso de la Ley $3 / 2007$, un target minorizador o individualizador ${ }^{2}$, en tanto que la rectificación registral del sexo constituye una posibilidad legal únicamente prevista para las personas transexuales $-\mathrm{y}$ no para todas, sino para las que acrediten esta condición en base a los criterios especificados en la ley-. La Ley 8/2014 aplica, por el contrario, un target universalizador a priori, en tanto que su redacción, configuración y ámbito de actuación se extiende al conjunto de administraciones públicas y, por ende, al conjunto de la sociedad. La ley comienza afirmando que la «existencia de personas transexuales es una realidad social presente desde los tiempos históricos más antiguos» (Ley 8/2014), por tanto, se evidencia una tendencia universalizadora y generalizada de la aplicación de esta ley. Cruells y Coll-Planas relacionan el modelo interseccional con el target universalizador, pues «se pone la atención en la relación de desigualdad teniendo en cuenta su vertiente estructural» (Cruells y Coll-Planas, 2013, p. 159). Por último, el target de la Proposición de Ley es universalizador, pues se pretende garantizar una igualdad social y evitar la discriminación a las personas LGTBI en diversos ámbitos como son el social, el sanitario, el familiar, el administrativo, el judicial, el educativo, el laboral, etc. La problemática toma como punto de partida el colectivo LGTBI, pero se dirige al conjunto de la

${ }^{2}$ La definición de un tipo de target $\mathrm{u}$ otro permite conocer si la política va dirigida a un grupo de personas concretas, o si por el contrario abarca un conjunto de población más generalizado. En el caso de las políticas LGTB, el target se analiza en función de si «las políticas contra la homo/transfobia se dirigen únicamente a las personas LGTB o bien al conjunto de la ciudadanía» (Cruells y Coll-Planas 158). 
sociedad con el objetivo de realizar una gestión igualitaria y libre de exclusiones con respecto a los grupos LGTBI.

Como se ha podido comprobar a lo largo de este trabajo, la incorporación de una perspectiva transversal e interseccional en las políticas públicas españolas en materia de igualdad y LGTBI no es una cuestión sencilla. Implantar los modelos y enfoques propuestos por la Comunidad Europea ha supuesto, y supone actualmente, un desafío de gran complejidad al que se enfrentan las instituciones y administraciones públicas de este país. Las reivindicaciones del movimiento feminista y de los colectivos LGTBI, acuciadas por la decisión firme y consolidada de romper con tabúes opresores, denunciar las desigualdades, condenar la violencia de género desde las calles y visibilizar sin miedo que otras identidades y sexualidades existen y son posibles, han contribuido a impulsar la elaboración de políticas públicas que incluyan, en el ejercicio de sus actuaciones, una perspectiva interseccional aplicada al colectivo LGTBI. Sin embargo, estas motivaciones no son suficientes, pues al tratarse de políticas que regulan aspectos tan delicados como las desigualdades de género y la configuración de identidades de género y orientaciones sexuales, resulta positivo que las personas con potestad para elaborarlas cuenten con formación específica sobre la materia y realicen sus propuestas desde una actitud empática con los colectivos discriminados a los que se pretende dar protección.

Contar con una ley de igualdad efectiva entre hombres y mujeres es un acontecimiento verdaderamente importante para los movimientos feministas y para los colectivos de mujeres. Al igual que esta ley fue necesaria en su momento y se obtuvo un resultado positivo con la promulgación de la Ley Orgánica 3/2007, para la igualdad efectiva de mujeres y hombres, las leyes que reconocen y amparan los derechos de las personas LGTBI también deben ser una tarea urgente para cualquier sociedad. A pesar de que muchos países a nivel mundial no tienen leyes LGTBI, como es el caso de España (a excepción de la Proposición de Ley LGTBI, que aún no está aprobada), es poco frecuente que las leyes existentes en materia de igualdad incluyan la orientación sexual y la identidad de género como otras formas de discriminación que dificultan el pleno alcance de la igualdad. Esto es lo que sucede con la LO 3/2007, anteriormente citada, y con la Ley $1 / 2010$, canaria de igualdad entre mujeres y hombres. Ambos textos dirigen sus esfuerzos a lograr la implantación de la transversalidad de género y a erradicar, por tanto, las desigualdades entre mujeres y hombres.

La ley canaria, tal y como se ha señalado anteriormente, se muestra proclive a atribuir valor a las diferencias como forma de contemplar la diversidad, pero sin desmarcarse de sus objetivos por la consecución de una igualdad en la acepción más académica y binarista del término. La transversalidad observada en las políticas estudiadas en este trabajo es una transversalidad que busca erradicar las desigualdades de género a través de las actuaciones de las administraciones públicas. A mi juicio se olvida, o simplemente se desconoce, que la efectiva erradicación de estas desigualdades conlleva también una atención y análisis de otros factores que la atraviesan y que influyen. Resulta poco eficiente crear una ley de igualdad como la LO 3/2007, con los esfuerzos no solo intelectuales, sino económicos y políticos que conlleva, que no dé muestras de querer conocer qué otros factores además del 
género pueden hacer que una mujer sea discriminada. En base a esto, se concluye que la tendencia de política pública es incorporar una transversalidad de género sesgada, en tanto que no es interseccional.

La cuestión de la equidad se encuentra estrechamente vinculada a este planteamiento, ya que parte de un concepto de justicia social que va a ser diferente según los grupos, precisamente porque la diversidad se constituye a través de diferencias de base. En mi opinión, la Ley 3/2007, reguladora de la rectificación registral de la mención relativa al sexo de las personas, no parte de la equidad, sino de buscar un cauce legal al que solo las personas transexuales que acrediten esta condición en las formas pertinentes pueden tener acceso. Con esta norma se construye un modelo de transexualidad parcial, que solo es válido si se cuenta con la acreditación profesional debida o si previamente la persona se ha sometido a una cirugía de reasignación. Si la cuestión del cambio registral se entendiera desde la equidad, esta ley tomaría como punto de partida un modelo trans* plural, abierto y fuera de la dicotomía masculino-femenino, con modelos diversos desde donde acceder, en condiciones de justicia social, a las garantías que en este caso la ley ofrece. Siguiendo la definición de Maingon y D'Elia sobre la equidad y la igualdad, podría afirmarse que la elaboración de esta ley constituyó un ejercicio de igualdad respecto al reconocimiento de derechos en comparación con otros reconocimientos legales. Es decir, cabe igualar en términos de reconocimiento la ley de rectificación registral a la Ley Orgánica 3/2007. La regulación de un aspecto tan importante para el colectivo trans* como es la modificación del sexo y nombre, y las desigualdades entre hombres y mujeres han obtenido amparo y protección legal, por tanto, se ha producido una situación de igualdad de hecho. Otra cosa distinta es, como ya se ha explicado, que esas leyes sean propiamente igualitarias en su alcance y objetivos. En relación con este tema, considero importante establecer diferencias entre la acción positiva y el concepto de diversidad. A mi juicio, se trata de términos totalmente contrapuestos, pues la acción positiva pretende igualar las condiciones del punto de partida para así combatir la desigualdad de género, mientras que la diversidad surge precisamente de esas diferencias, y por tanto no es posible equipararla a la acción positiva. Las condiciones de partida diversas, así como las que existen dentro del colectivo LGTBI, son necesarias para el desarrollo de políticas interseccionales, pues es justamente esa diversidad la que hace que existan y tengan sentido.

Como ya se adelantaba en epígrafes anteriores, la cuestión de las categorías de identidad genera un debate desde ámbitos como la sociología o la filosofía, encontrando también un espacio de discusión en las teorías feministas y queer. Las preguntas que aquí surgen son estas: jes posible elaborar una interseccionalidad que supere estas categorías?, ¿tendría sentido una teoría interseccional sin etiquetas? En mi opinión, la clasificación de las opresiones que históricamente han afectado y siguen afectando al conjunto de la humanidad es necesaria. En primer lugar, porque es importante saber de lo que se está hablando para actuar sobre ello, y, en segundo lugar, porque categorizar significa nombrar, y nombrar significa visibilizar. Si una mujer lesbiana se nombra a sí misma como lesbiana, y entiende el significado cultural, político y social del término, podrá hacerse con las herramientas necesarias para luchar, siendo consciente de su categoría, contra posibles discriminaciones y 
rechazos que como mujer lesbiana es susceptible de sufrir. En relación con la interseccionalidad de las políticas, considero que no puede concebirse una aplicación interseccional que no contemple grupos clasificados y «etiquetados» según su pertenencia a un colectivo discriminado. Si no se identifican las causas que intervienen en una situación de desigualdad ni se investigan las razones estructurales que subyacen a ella, la aplicabilidad de la perspectiva interseccional no tiene sentido alguno. Es precisamente la existencia de las categorías sociales, de raza, de orientación sexual e identidad de género, de procedencia geográfica, de clase social, etc., las que posibilitan un análisis de las opresiones desde un punto de vista de la interrelación entre muchos factores. Pero a veces la categorización puede producir estigmatización, y es por ello que existe una delgada línea entre la clasificación de grupos con fines de análisis y la utilización de las categorías como forma de señalamiento. Un ejemplo es lo que ocurre con el término "feminista», cuando es utilizado por grupos opresores y contrarios al movimiento con una intención discriminatoria y ofensiva. Esto produce la autoinvisibilización por parte de quien se considera feminista respecto a un grupo de personas ante las que prefiere no reconocerse como tal, precisamente por el miedo a pronunciar el término y ser testigo de ciertas tensiones. En lo que respecta a las políticas interseccionales, es necesario conocer este límite entre las dos prácticas (positiva y discriminatoria), para no cometer el error de definir una política pública que nazca del deseo de reconocer los derechos de un grupo oprimido, y que finalmente genere una visión estigmatizante hacia el mismo. En mi opinión, esto es lo que sucedió con la Ley 3/2007, relativa a la rectificación registral del sexo. El grupo diana al que se reconoció el derecho a modificar su nombre y sexo fue el colectivo trans*, pero de la redacción del texto y de la aplicación de este, se derivaba un modelo de transexualidad entendido desde la patologización y el binarismo. Es evidente que no es tarea fácil hacer política pública sobre un grupo concreto y no caer en la estigmatización, pero creo que, con los conocimientos y recursos adecuados, es posible avanzar hacia una regulación justa que represente de forma digna los anhelos y realidades de estos colectivos.

Por otra parte, la confusión de la interseccionalidad con las políticas sectoriales supone un grave perjuicio para la consecución de políticas públicas con un enfoque integrado. Por ejemplo, regular la asistencia a mujeres en situación de violencia de género sin vincular a esto factores como la racialización, el nivel educativo, económico, etc., y, además, elaborar políticas que traten de forma independiente estas realidades, dificulta en gran medida la identificación de las necesidades y la posterior elaboración de políticas con una visión transversal.

\section{CONSIDERACIONES FINALES}

Del estudio de la Ley 3/2007, reguladora de la rectificación registral, se desprenden varias conclusiones. La primera pasa por observar una contradicción relativa al requisito de la cirugía de la reasignación. Por una parte, el art. 4.2.2 establece que «no será necesario para la concesión de la rectificación registral de la mención del sexo de una persona que el tratamiento médico haya incluido cirugía de reasig- 
nación sexual» (Ley $3 / 2007$, art. 4.2.2). La eliminación de este requisito, que, hasta la fecha, y como se ha observado, era conditio sine qua non para que una persona fuera jurídicamente considerada transexual, manifiesta, a priori, una ruptura con el sistema binario del género y con el planteamiento biologicista que entiende la genitalidad como determinante del género. En base a ello, parece que se abre un nuevo espectro de posibilidades que acepta la existencia de identidades fuera de los moldes del binarismo. Pero por otro lado la ley se contradice, pues la disposición transitoria única exime de los requisitos de la condición de transexualidad (informe, ausencia de trastornos mentales y tratamiento continuado) a quienes certifiquen haber sido sometidos a la cirugía de reasignación en un momento anterior a la aprobación de la norma. Esto supone abandonar la tendencia antibinarista que se observaba en el art. 4.2.2, para volver a caer en la concepción biologicista que entiende que la persona es transexual en tanto que ha pasado por ese proceso de reasignación quirúrgica. Por todo ello, esta ley propicia que exista desigualdad dentro del propio colectivo trans*, en tanto que estos dos preceptos establecen una doble vara de medir que utiliza el requisito de la cirugía de reasignación según convenga. En términos de interseccionalidad, esta contradicción afecta de manera negativa a su consecución, ya que la relación entre el género y la transexualidad se ve limitada por la merma de posibilidades de desarrollo en un abanico de identidades existentes fuera del binarismo de género hombre-mujer. Por tanto, no existe interseccionalidad, pues la ley establece unos únicos requisitos aplicables a un único modelo de persona trans* sin tener en cuenta la diversidad en el propio colectivo, una diversidad que alberga a personas que han sido sometidas a la cirugía de reasignación y a otras que no la han realizado, pero que tienen igualmente clara cuál es su identidad de género y el derecho para considerarse trans*.

Como segunda conclusión, no se puede afirmar que la Ley 3/2007 aplique una perspectiva interseccional en tanto que no aborda la intersección de otros factores, que, debido a las características y objetivos de la ley, deben contemplarse. Es el caso de los menores y las personas extranjeras trans*, que no son incluidos como sujetos legitimados en el art. 1, por tanto, se les niega el reconocimiento de un derecho importante como es el cambio de nombre y sexo en el registro civil. Tampoco se contempla que puedan acogerse a esta norma las personas intersexuales para cambiar el sexo «hombre» $\mathbf{o}$ «mujer» que conste en sus documentos oficiales por el de «intersexual».

En segundo lugar, del análisis de la Ley 8/2014 se extraen una serie de consideraciones. Se presenta como una norma que actúa en pro de la pluralidad, entendiendo que dentro del propio colectivo trans* existe una diversidad de comportamientos, lo que da lugar a que la respuesta de las administraciones públicas se articule a través una atención integral y centrada en las circunstancias del caso concreto. Además, es consciente de la influencia de otros factores que pueden agravar la situación discriminatoria de base. A pesar de la aparente tendencia interseccional que se desprende de las primeras páginas del texto, lo cierto es que, de forma similar a la anterior ley, existen contradicciones que dificultan su identificación con una política pública de carácter interseccional. Estas contradicciones se explican, por una parte, por la autodeterminación consciente de la identidad de género, y por otra, 
por la definición de "persona transexual» que se detalla en el artículo 2. Resulta incoherente mostrarse a favor del derecho de autodeterminación y exigir a la vez una serie de requisitos que acrediten la condición de transexualidad. Estas condiciones se adoptan de la Ley $3 / 2007$, aunque se elimina el requisito del tratamiento.

Otra de las razones que hacen que no se aplique la teoría interseccional en esta norma tiene que ver con la forma en que otros factores discriminatorios son integrados y vinculados con la transexualidad. Así, hay ciertos artículos que fomentan la empleabilidad de las personas trans* y procuran una atención integral a mujeres trans* en situación de violencia de género. Sin embargo, estas situaciones discriminatorias no se incorporan de forma estructural en el análisis que la norma realiza a fin de asegurar su objetivo: la no discriminación por motivos de identidad de género.

En tercer lugar, del análisis de los criterios de interseccionalidad aplicados a la Proposición de Ley LGTBI se desprende que, de las tres políticas analizadas, esta es la que en mayor medida se aproxima al modelo de política pública interseccional. Así, dedica algunos preceptos a explicitar los factores que provocan una discriminación múltiple, y además amplía las medidas de no discriminación a colectivos doblemente vulnerables que, por características relacionadas con la edad, diversidad funcional u orientación sexual, son proclives a que se generen situaciones de discriminación de carácter estructural. En cuanto al derecho de autodeterminación citado en las anteriores leyes, es preciso señalar que esta Proposición de Ley, en ningún momento pretender condicionar la existencia de la transexualidad a informes ni documentos sanitarios acreditativos, pues entiende que se trata de una cuestión que tiene que ser autodeterminada por la persona y así lo reafirma en los artículos referidos a las personas trans*, desvinculándose de la visión patologizante observada en leyes anteriores.

Los esfuerzos que se advierten en esta Proposición de Ley por ahondar en las múltiples causas y factores que hacen que un colectivo sea discriminado se ven apoyados en la exposición de motivos por su marcada vocación integral, relacionando este concepto con «la regulación de los derechos en todos los ámbitos sociales: familia, sanidad, educación, deporte, cultura y ocio, justicia y seguridad, medios de comunicación, protección social y laboral y relaciones con las Administraciones Públicas» (Proposición de Ley LGTBI, 2017). Que el objetivo de esta Proposición legislativa sea erradicar las desigualdades que sufre el colectivo LGTBI a través del reconocimiento de sus derechos en todos los ámbitos de la vida social no es exactamente lo que persiguen las políticas interseccionales. En mi opinión, partiendo de la buena disposición que muestra esta norma hacia la integración de la diversidad, debería ir un poco más allá y proteger al colectivo LGTBI ahondando en las causas estructurales e interconectadas que provocan su discriminación.

La existencia de políticas públicas cuyos objetivos son la aplicación de la transversalidad de género en el conjunto de actuaciones de los poderes públicos es una realidad que desde las últimas décadas va en aumento. A partir de los mandatos europeos de incorporar la perspectiva del mainstreaming a la política pública, han sido muchas las leyes que han sido elaboradas para dar cumplimiento a dicha orden. Ejemplos de esto estudiados en este trabajo han sido la Ley Orgánica 3/2007, para la efectiva igualdad entre mujeres y hombres, y la Ley $1 / 2010$, canaria de igualdad, 
entre otras. Ahora bien, ¿̇tiene aplicación la interseccionalidad en las políticas, y de forma más concreta, en las leyes de identidad de género analizadas? En base a los instrumentos legales objeto de análisis, afirmar esto ya es una cuestión más compleja. Según lo estudiado, se puede concluir que la teoría interseccional conlleva un proceso más lento en la aplicación e integración en las normas que se elaboran. No obstante, se observa una tendencia favorable al reconocimiento de derechos de grupos históricamente oprimidos desde una posición interseccional. Sin embargo, la política pública de nuestro país se encuentra todavía un poco alejada del ideal que la teoría interseccional pretende conseguir.

En cuanto a la cuestión de si la interseccionalidad en las políticas LGTBI ha tenido un recorrido más amplio y desarrollado en las comunidades autónomas (concretamente en Canarias) que a nivel estatal, es preciso establecer ciertos matices. Si bien es cierto que la primera ley que reguló algún aspecto relacionado con la transexualidad fue la Ley $3 / 2007$, reguladora de la rectificación registral de la mención relativa al sexo de las personas, su contenido ha quedado obsoleto a nivel social -que no legal, pues sigue en vigor-. Los colectivos trans* reclaman una regulación que no los discrimine por razones de binarismo y de biologismo, y que por tanto sea interseccional dentro del propio colectivo. Consecuencia de ello, existe la Proposición de Ley Trans*, texto que pretende regular el derecho a la libre determinación de la identidad y expresión de género, y que es elaborado en la línea cuasiinterseccional procedente de la Proposición de Ley LGTBI. Por tanto, puede concluirse que el desarrollo de políticas interseccionales LGTBI por parte del Estado es cada vez mayor y más concienciado acerca de la vulneración de derechos que supone no ser reconocido como sujeto de derecho por motivos de identidad de género u orientación sexual. En el caso de Canarias, a juzgar por la ley vigente en esta materia, la Ley 8/2014, no se concluye que esta norma pueda representar de manera sólida los criterios que determinan la teoría interseccional, dadas las contradicciones que experimenta en su articulado, como se ha visto anteriormente. Se puede afirmar que en este caso Canarias actúa un paso por detrás del Gobierno central, pues recientemente se ha aprobado en el Parlamento una Proposición de Ley de igualdad social y no discriminación por razón de identidad de género, expresión de género y características sexuales, cuyos objetivos principales son «la despatologización de las realidades trans y la defensa de la autodeterminación del género» (https://www.canarias7.es/), algo que ya adelantaba la Proposición de Ley Trans* del año 2018.

En conclusión, entiendo que la falta de información y la poca visibilidad que históricamente ha tenido el colectivo trans* ha derivado en la estigmatización por parte de las instituciones públicas, y por tanto de la sociedad. Esto se traduce en la regulación sesgada de los derechos de las mujeres y de las personas LGTBI, donde ni los términos empleados ni los razonamientos discutidos ni las soluciones aportadas son las más adecuadas, consecuencia del concreto momento sociohistórico, político y cultural en que se fraguan las políticas. Sin embargo, la evolución de las teorías queer y la consolidación de las teorías interseccionales apuntan hacia la configuración de políticas de igualdad y LGTBI que incorporen cada vez más una perspectiva de tratamiento de las desigualdades con un enfoque integrado, multifactorial e interconectado. Solo así será posible profundizar en las verdaderas causas 
de exclusión, causas que no surgen de la inexplicable estigmatización de un grupo, sino que se originan por la combinación de otras motivaciones que comparten la misma base estructural de opresión. 


\section{BIBLIOGRAFÍA}

\section{Normativa}

Declaración y Plataforma de Acción de Beijing. (1995). Naciones Unidas. Recuperado de https://www.unwomen.org/-/media/headquarters/attachments/sections/csw/bpa_s_final_ web.pdf?la=es\&vs=755.

Unión Europea. Directiva (UE) 95/593/CE del Consejo, de 22 de diciembre de 1995, relativa a un programa de acción comunitario a medio plazo para la igualdad de oportunidades entre hombres y mujeres (1996-2000) L 335 de 30/12/1995 pp. 0037-0043.

\section{Documentos oficiales (PLANEs, Estrategias)}

Plan Estratégico de igualdad de oportunidades (2008-2011). Ministerio de Igualdad. Recuperado de http://www.mitramiss.gob.es/ficheros/ministerio/igualdad/Plan_estrategico_ final.pdf.

Gobierno de Canarias. Consejería de Presidencia, Justicia e igualdad. Estrategia para la igualdad de mujeres y hombres 2013-2020. Recuperado de https://www.gobiernodecanarias.org/cmsgobcan/export/sites/icigualdad/_galerias/ici_documentos/documentacion/Planes/Estrategia_ Igualdad_2013-2020.pdf.

\section{BiBLIOGRAFÍA CITADA Y CONSULTADA}

Bilge, S. Théorisations féministes de l'intersectionalité. Diogène. Presses Universitaires de France, 2009.

Brah, A. Cartografia de la Diáspora: identidades en cuestión. Traducción Sergio Ojeda. Madrid: Traficantes de sueños, 2011 (1996).

Bustelo, M., Lombardo, E., et al. «Policy Frames and Implementation Problems: the Case of Gender Mainstreaming»/ MAGEEQ (Mainstreaming Gender Equality). Proyecto finanzialo por el V Programa Marco de Investigación de la Unión Europea, MAGEEQ (HPSE-CT-2002-00127), 2003-2005.

Coll-Planas, G. (coord.). Combating Homophobia. Local Policies for Equality on the grounds of Sexual Orientation and Gender Identity. A European White Paper. Barcelona: Ajuntament de Barcelona, 2011.

Cruells, Marta y Gerard Coll-Planas. "Challenging equality policies». The emerging LGBT perspective. European Journal of Women's Studies, 20: (2013) pp. 122-137.

Cruells, M. Tesis doctoral. La interseccionalidad politica: tipos y factores de entrada en la agenda política, jurídica y de los movimientos sociales. Barcelona: Universidad Autónoma de Barcelona, 2015.

Cubillos, J. La importancia de la interseccionalidad para la investigación feminista. Madrid: Oxímora Revista internacional de ética y política, 7, (2015) pp. 119-137.

Domínguez, C., Forest, M., y SÉNAC, R. Quépoliticas para qué igualdad. Debates sobre el género en las politicas públicas en Europa. Valencia: Tirant Humanidades, 2013. 
García, E. Politicas de igualdad, equidady Gender Mainstreaming. ¿De qué estamos hablando? Marco conceptual. Fondo España-PNUD y Agència catalana de Cooperació al Desenvolupament, 2008. Recuperado de http://www.americalatinagenera.org/es/documentos/doc_732_Politicasdeigualdad23junio08.pdf.

Lombardo, E. y Verloo, M. «Institutionalising intersectionality in the European Union?». Policy Develompments and Contestations, International Feminist Journal of Politics, 4 (2009), pp. 478-495, 2009.

Maingon, T. y D’Elia, Y. La equidad en el desarrollo humano: estudio conceptual desde el enfoque de igualdady diversidad. Documentos para la discusión. Informes sobre desarrollo humano en Venezuela. Venezuela: Programa de las Naciones Unidas para el Desarrollo, 2004.

Martínez-Palacios, J. Exclusión, profundización democrática e interseccionalidad. Investigaciones Feministas, Madrid: ediciones complutense, 2017. Recuperado de https://revistas.ucm.es/ index.php/INFE/article/view/54827/51189.

Platero, R. Lucas Transexualidad y agenda politica: una historia de (dis)continuidades y patologización. Politica y sociedad, 46:1, (2008), pp. 107-128.

Platero, R. Lucas «¿Son las políticas de igualdad de género permeables a los debates sobre la interseccionalidad? Una reflexión a partir del caso español». Revista del CLAD Reforma y Democracia, 52 (2012), 135-172.

Rawls, J. Teoría de la Justicia. Fondo de Cultura Económica de Argentina, Buenos Aires, 1979.

Real Academia Española: Diccionario de la lengua española, 23. a ed., [versión 23.3 en línea] https://dle.rae.es [12 de abril de 2020]. 